\title{
Online Cultural Transmission: A Memetic Approach to The Case of Tombili The Cat
}

\author{
Tekrin D. ${ }^{1}$ \\ ${ }_{1}^{1}$ Dilara TEKRIN, (Turkey) \\ e-mail: dilaratekrin@gmail.com
}

\begin{abstract}
A meme is a unit of information that is capable of spreading from an individual's brain to another, and through this transmission the meme replicates itself. Richard Dawkins has introduced the meme in 1976 as "a unit of cultural transmission". After Dawkins, numerous scientists have taken an interest in the concept of meme. The definition of meme and its examples have been a much-debated topic. As interesting as the concept of meme is the process of how a meme replicates and leaps among people's memories. Memetic science aims to clarify these points and thus studies how memes replicate, spread, evolve, and how cultural transmission occurs. The introduction of memes and the development of memetics had arisen before the Internet became available in households and social media platforms turned into everyday practice. Today in the age of the digital transformation, the study of memes becomes more intriguing since the viral content and the Internet memes are among the most debated digital phenomena. This paper attempts to answer if Internet memes can be considered as units of cultural transmission. Are they a digital version of memes, or do they have distinct functions? I believe answering these questions will contribute to the studies of Internet memes and will provide an insight into how digital technologies impact cultural transmission processes. I am going to review the major theories and former studies on memes including the topics; the functions of meme replication, the survival value and the selection criteria of memes. Apply these theories to Internet memes in order to understand whether Internet memes can be considered as a digital version of memes or not. Analyze the Turkish Internet phenomenon Tombili the Cat as a case study.
\end{abstract}

Keywords: Meme, Memetics, İnternet Meme, Cultural Transmission

\section{Introduction}

This chubby cat lounging on the pavement as if sitting on a comfy chair is Tombili the Cat, with whom the active users of social media are closely acquainted. Tombili is a famous street cat whose fame has reached beyond national borders, who was in news coverage even after he died, in whose name a statue was built and whose statue has taken a place in İstanbul city guides (url1, 2019) And moreover he is a world-renowned Internet meme (url2, 2019). Although an internet meme is usually known as an image and text written over the image, it is a concept based on a complex literature. The origin of this entertaining, funny, popular cultural element is based on the concept of "meme" introduced to the scientific world by evolutionary biologist Richard Dawkins as a unit of cultural evolution. Meme and memetic science are a concept that concerns so many disciplines that numerous scientists from many different fields, such as cognitive science, psychology, sociology, cultural anthropology, biology and communication work on this concept. Despite the fact that the word meme has a very short history dating back to only 1976. 


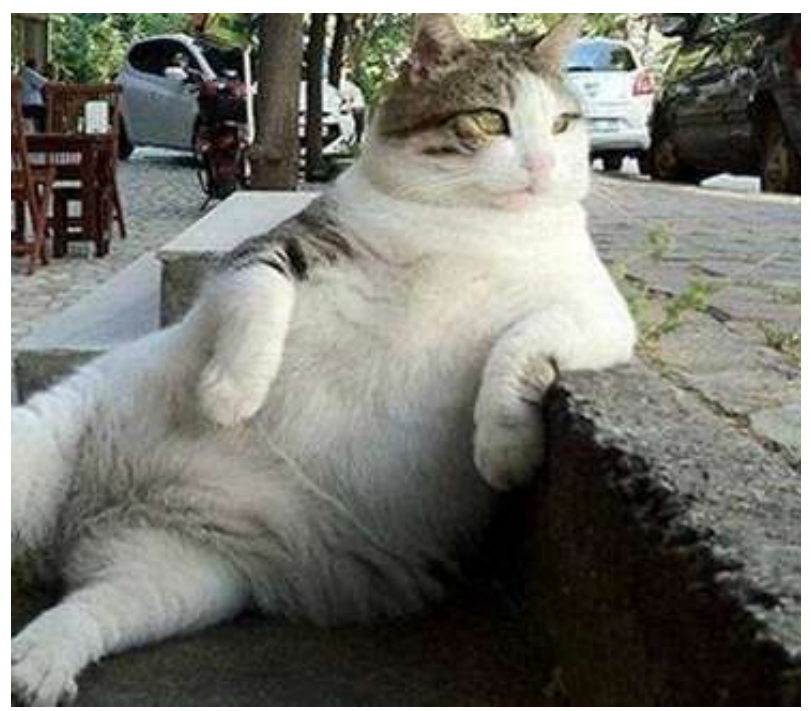

Image 1. Tombili the Cat

This research approaches the connection between the concept of meme -which is ambitious, not very easy to define and understand- and the Internet memes via the famous Tombili meme. As the name implies Internet memes are based on the concept of the meme. However, this study aims to clarify whether the Internet memes are only a digital version of memes or did digital transformation provide them new possibilities and functions?

To this end, we will first analyze the definition of meme, its decisive features and then examine theories regarding how and why memes replicate and continue with applying them on the case of Tombili the Cat.

\section{The Concept of Meme}

Oxford Dictionary gives two definitions for the word "meme" whose name we come across more and more every day with an increased momentum in the last ten years: "1. An element of a culture or system behaviour passed from one individual to another by imitation or other non-genetic means. 2. An image, video, piece of text, etc., typically humorous in nature, that is copied and spread rapidly by Internet users, often with slight variations." While the first definition shows affiliations with the meme definition introduced to the literature in 1976 by Dawkins, the second definition belongs to the Internet memes that we will be examining in detail below. In the last chapter of his significant work about genes The Selfish Gene, the renowned biologist introduced the concept of meme through an analogy with genes. He claimed that memes are the second replicator kind on our planet and advocated that just as the genes are the building blocks of evolution, memes carry out the cultural evolution (Dawkins, 1976). Dawkins combined the Greek words "mimeme" (imitation) and "gen" (gene) to form the English word "meme." As it is quoted almost in every text regarding memes, the first examples Dawkins offered for memes were as follows: Tunes, ideas, catch-phrases, clothes fashions, ways of making pots or of building arches (Dawkins, 1976). However, in the year 1976 when Dawkins provided these examples, mass media was still continuing to dominate and digital communication had not yet become a part of our daily practice. In light of this information, it can be assumed that Dawkins would include Instagram influencers, selfie trends or the Kardashian family in these examples if he was to introduce the concept of meme in today's world.

To comprehend Dawkins' concept of meme, we need to look into the term "replicator" from the biology literature. Meme as a replicator means that they propagate from one individual's mind to the other by means of multiplying and replication. One of the first examples of memes given by Dawkins is catch-phrases. When a catch-phrase is heard and repeated it sticks in someone else's mind. By repeating it, the new host of the catch-phrase enables the circulation of the catch-phrase in the same way. In theory, the chain of propagation can continue until all the minds in the world have a copy of this meme (of the catch-phrase in this example). The crucial point that is of great importance in this overall chain is that every catch-phrase will not be repeated just as every encountered post on social media is 
not shared with the followers. Dawkins states that competing memes are in an eternal race to take place in our minds and the memes with high "survival value" complete the race as winners (1976). Songs, phrases, ideas that have high survival value become parts of the culture by continuing to live in people's minds while the others fade away.

Dawkins was not the only scientist who studied units such as memes that multiply by replication and enable cultural evolution. K. Eric Drexler's term "mental replicator" and Lumsden and Wilson's "culturgen" term have also taken their places in the literature, not dating far apart from each other (Moritz, 1990). By the 90s, memes had become a concept researched by numerous scientists. Francis Heylighen, who holds a prominent position in these researches, focused on the stages of meme replication and defined memes as units of information in an individual's mind that can be replicated in another's. According to Heylighen, anything that can be learned or remembered (ideas, knowledge, habits, skills, images) can be a meme (1999).

Another significant name that contributed the literature regarding memes is Susan Blackmore. In The Meme Machine, Susan Blackmore not only defines memes as new replicators following her pioneer Dawkins, but also claims that memes are responsible for a number of situations from our brains being larger than our far ancestors to the concept of family in modern times. Furthermore, she advocates that the function of language is to spread the memes (Blackmore, 2011). According to Blackmore memes are "regulations to conduct behaviors, they are preserved in minds (or in other objects) and they are transmitted by imitation." While Plotkin defines this highly significant but challenging concept as "an internal representation of information" (Brodie, 2014:29) and Dennett defines it as "a complex thought that turns itself into a form of a memorable unit" (Brodie, 2014:31), Brodie states that "a meme is a unit of information in a mind whose existence influences events such that more copies of itself get created in other minds" and claims that the memetic science is such a significant development that it can even cause a paradigm shift in the science of thought (Brodie, 2014:34). The common feature of these definitions is that memes spread from one's mind to the other and conduct a cultural communication in this process. This definition of meme sets the most efficient definition for this research as well.

\section{How Much Is a Meme Unit?}

Among the difficult questions regarding memes, "What is the measure of a meme unit?" comes first. If we put this question in practice, one of the most helpful examples would be to ask how much of a song that is stuck in our minds can be considered as a meme by itself. Dawkins explains his approach to this subject via an analogy with genes and suggests that if a gene "is a length of chromosome with just sufficient copying-fidelity" then memes can be defined as such. Dawkins gives Beethoven's Ninth Symphony as an example. "If a single phrase of Beethoven's Ninth Symphony is sufficiently distinctive and memorable to be abstracted" then according to Dawkins "it deserves to be called one meme" (1976). Thus, the first phrase of the Ninth Symphony, which sounds familiar to everyone, can be defined as a meme. Dennett adapts a similar approach to Dawkins' in defining meme units stating "memes are the smallest units that replicate themselves with reliability and fecundity" (Brodie, 2014). On the other hand, Blackmore suggests that a meme unit cannot have a precise definition, giving jingles and books as examples. She underlines that copyrights can be bought regardless of a unit criterion and any unit that can be considered original enough to have a copyright can be considered as a meme (Blackmore, 2011: 71).

\section{Survival Value of Memes}

Certain information, thoughts, ideas and images become memes and some just fade away. What makes the difference between these two groups, why some of them "succeed" to take their places in minds and become part of the cultural milieu? What is referred to by the phrase successful meme-a phrase that we come across frequently in literature regarding memes-? There is one basic answer that can be given to these questions. The success criteria of a meme rely on the number of minds it spreads to. Memes that can reproduce by replicating themselves in a high number of minds have high survival values, meaning they are successful memes. While their competitors fade away, successful memes take their places in minds, get replicated rapidly and become a part of the culture. Dawkins draws attention to the fact that such as the successful genes, successful memes have three distinctive properties: Longevity, fecundity and copying fidelity (1976). 
"Longevity" underlines the importance of the meme's preservation in the mind for a long period and thus being able to have the opportunity to be replicated. For instance, the chances of a melody that can be forgotten in just minutes being spread to other minds will be lower compared to a memorable melody. In addition to this, if we consider that a meme has a lot of replications, the meme will be as long living as the number of copies it has. To be forgotten in one of the hosting minds, to come to its life's end will not have such importance in the survival trial. In this context, the significance of the replication frequency of the meme, the second criteria deemed necessary by Dawkins for having a high survival value, becomes apparent. "Fecundity" means the ability of the meme to replicate itself. Any information that cannot be replicated will not be able to leap from one mind to another. "Copying fidelity" is about the error-free copying of the meme. Dawkins draws attention to copying fidelity as a debatable subject since certain memes go under changes when replicated. One of the most accurate examples of it would be the case of the urban legends. Urban legends, which go through minor changes every time they are told, still continue to spread to different minds despite these "copying errors" and Dawkins defines this situation as "perpetual mutation and blending" (1976). Therefore, the combination of these three properties -longevity, fecundity and copying fidelity- determine the meme's success in replicating itself in one mind after another while others fade away. Below we will analyze which of these properties are present in the example of the Tombili the Cat.

\section{How Do Memes Reproduce?}

Memes reproduce by replication but how does this process take place? Dawkins' criteria of longevity, fecundity and copying fidelity explained the criteria that determine the memes' survival values and which memes will be replicated and spread. Another prominent researcher in the memetic studies, Francis Heylighen's model of four stages of meme replication analyzes the selection stages the meme goes through when being replicated: Assimilation, retention, expression and transmission. A meme that passes these four stages becomes successful (1999).

The first stage of meme replication is "assimilation." If the meme is noticed, understood and offered a place successfully in an individual's mind it gets assimilated. In order to be assimilated the meme has to attract attention. To be understood and offered a place in the host's mind the meme has to enter the host's cognitive system. At this stage, for a successful meme replication, the meme should be relatable to the information and other memes that are already available to the individual's mind. For example, this stage assumes that if an urban legend regarding vampires is to draw attention and is to be accepted by the individual's cognitive system, preliminary information about vampires should be present in the mind of the individual and the individual should have heard previous stories about vampires.

A meme that has passed the assimilation stage and got accepted to the mind should have a certain "retention" period in order to continue its replication journey in other minds. We cannot speak of a possible meme replication if the meme is immediately forgotten after the assimilation stage. In the retention stage, factors such as being interesting, arousing a feeling and being frequently repeated against being forgotten can be considered decisive. For example, we can assume that an exciting, moving catch-phrase will stay in the mind longer than a catch-phrase that doesn't evoke any emotion. In addition to this, the longer a meme's retention period in the mind without being forgotten the more chances it will have to move to the third stage, which is expression.

If the meme is deemed valuable enough by the host to transmit to others it will complete the third stage of replication, "the expression stage" successfully. While some memes can be interesting enough for assimilation and retention, the individual may shy away from sharing it; he/she may think it will not be interesting for others. In that case the meme will not be able to achieve the third stage of replication and its existence will come to an end when it is forgotten by the individual. A meme which is considered valuable enough by the host to be expressed moves to the last stop of the replication process: Transmission.

The transmission of a meme from one mind to another requires a sufficient meme medium. The number of examples such as of books, photographs and works of art have increased rapidly with the help of digital technologies. Heylighen states that there can be possible selection reasons for the message to get corrupted before reaching the receiver or for the losses that can occur during the transmission. 


\section{Internet and Memes}

Blackmore advocates that all communication tools help memes spread and they were produced with this purpose in the first place (2011). As communications tool developed and their meme transmission capacities increased, the dissemination of memes have also accelerated and expanded. Even this point of view is enough to clarify by itself how significant Internet technology is for memes. During the process that started with the invention of writing, communication tools had various impacts on the dissemination of knowledge and the circulation of memes.

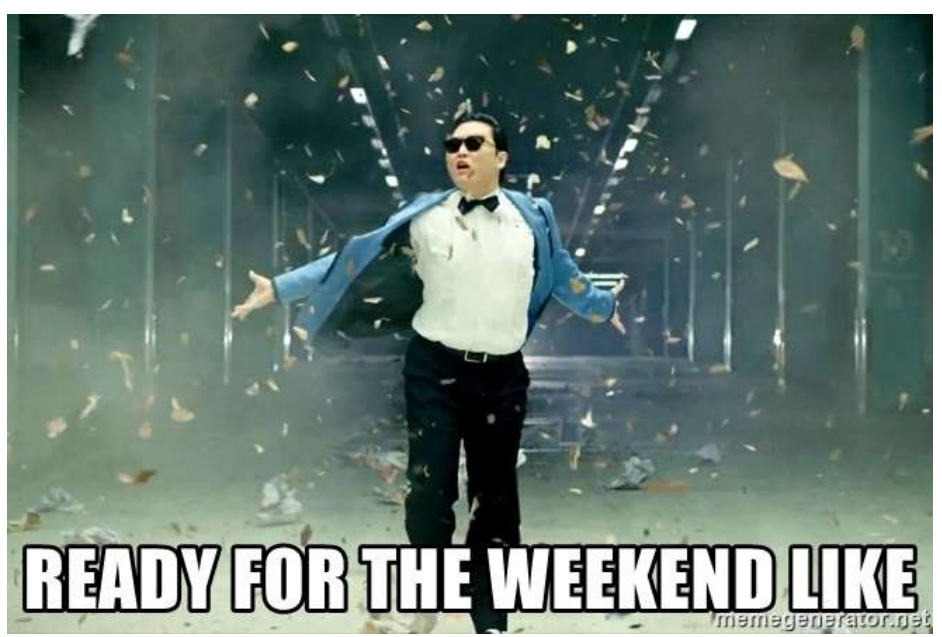

Image 2. An Image Macro Example

If we compare the pre-printing era when the books were manually copied and today's world when digital publications can reach thousands of people in just seconds, we can assume how much speed meme transmission has gained through the history of communications. Although the invention of printing and mass media tools were important developments for memes, we have not come across concepts such as "TV meme" or "magazine meme", whereas the concept of "Internet meme" was introduced to our lives in 2000s and have become one of the trademarks of popular culture. Even this is enough to reveal the significance of digital transformation as a milestone for memes. In this part, first we will look into the definition of the Internet meme and then focus on analyzing meme theories via the Internet meme Tombili The Cat, to comprehend what types of transformation the Internet brought on to memes.

\section{Internet Meme}

The definition of Internet meme given by the Oxford Dictionary: An image, video, piece of text, etc., typically humorous in nature, that is copied and spread rapidly by Internet users, often with slight variations. Consisting of an image and an accompanying text, known as image macro in English and "caps" in Turkish, such as the content we see in the images presented in this research, is the first format that comes to mind regarding Internet memes. However, anything that is transmitted on the Internet-from videos to lyrics and gifs-can be regarded as Internet memes.

It is important to note that there have been disagreements regarding its definition among the people studying Internet memes. In order to make the distinction between a viral content and an Internet meme, Börzsei underlines the susceptibility of the meme to alteration as stated by Dawkins under the subject of copying fidelity. While Börzsei includes YouTube videos, marketing contents and music videos that do not go through any changes in viral contents group, she claims that internet memes go under a change during every transmission and if not they cannot be defined as Internet memes (2013). With a similar approach Goriunova also distinguishes between organic memes and marketing memes, and argues that contents that naturally spread and go under a change or are mutated can be considered as Internet memes (2013). These claims conflict with the meme definition provided by Dawkins, 
Blackmore and other pioneers of memetics but it is important to keep in mind that while these initial definitions were being made the Internet memes had not yet come to existence.

\section{The Case of Tombili The Cat}

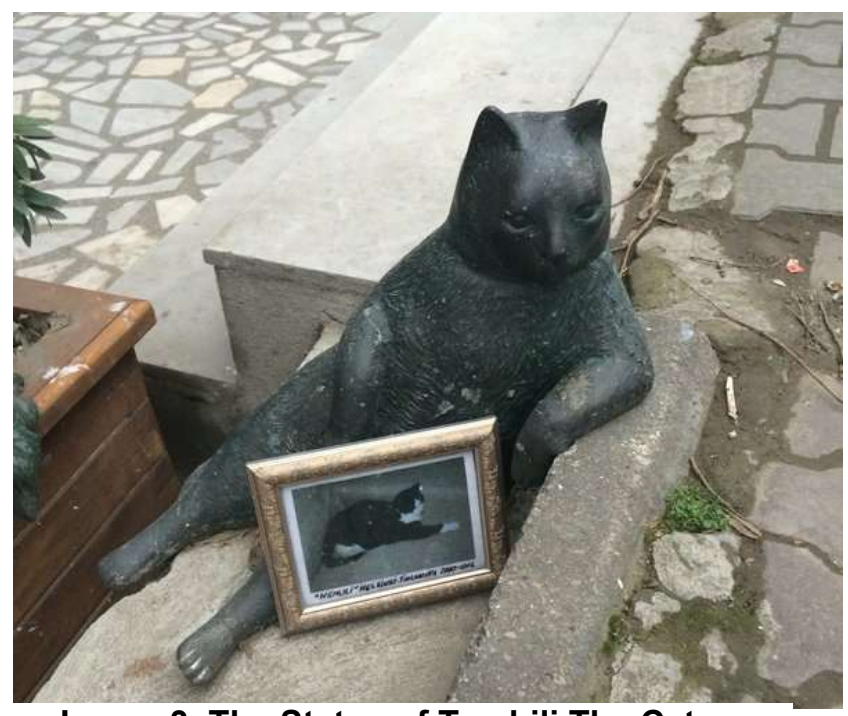

Image 3. The Statue of Tombili The Cat

Tombili The Cat is a stray cat born in a district alive with its street culture. Residents of this area do not talk about Tombili without mentioning especially how fat and cranky he is (url3, 2019). As it is the custom in street culture Tombili is an animal loved, adapted, fed and taken care of by all the residents of the neighborhood, not just one individual. One of Tombili's fans has photographed his peculiar sitting position and shared the photo on social media in a short time period, Tombili The Cat has turned into a social media phenomenon and became a cat with an international fame. In fact, Tombili The Cat, who lost his life after an illness, is so famous that his story has even appeared in foreign media. He also has a dedicated page on a meme platform known as Knowyourmeme (url4, 2019). Residents of the neighborhood submitted a petition to build his statue after his death and the municipality answered this request by getting his statue built and placed on the same spot his photo was taken - the photo that turned him into an Internet phenomenon. Tombili's statue also had broad media coverage on the Internet and many people shared the photo of the statue on social media. However, one night the statue was mysteriously stolen. This incident also had broad media coverage (url5, 2019) and had repercussions on social media. One morning the residents of the neighborhood saw that the statue of Tombili has come back yet again mysteriously. Tombili's story which has continued even after his death made him even more famous and as the image macros that have accompanied every stage of his story were shared on social media, his fame has reached all parts of the world.

\section{Dawkins' Survival Value and the Example of Tombili The Cat}

Richard Dawkins mentioned that genes with high survival value have three common properties namely longevity, fecundity and copying fidelity. He claimed that these properties have not exact but similar functions in terms of memes as well. With the hypothesis that the properties that can vary between memes and genes can also vary in Internet memes, I will interpret the criteria of longevity, fecundity and copying fidelity via the example of Tombili The Cat.

\section{Longevity}

The first property required for genes and memes to have a high survival value is longevity. This is based on the idea that the memes with the most longevity are more memorable, are expected to have more opportunities of replication. Dawkins also underlined that by longevity he refers to the total lifespan of all the copies as opposed to the lifespan of one particular copy. If we consider that every time an Internet meme is shared it takes place in a high number of feeds on social media plat- 
forms, we can conclude that every share of the meme adds more to its lifespan. Every time a content about Tombili is shared, new copies are replicated and these copies, by recurring in high volume on people's social media feed, spread numerous copies of the meme that have the potential longevity provided by digital technology.

\section{Fecundity}

The second prerequisite of high survival value, fecundity, can also be regarded among the properties taking advantage of the benefits provided by digital communication. Social media platforms that enable the replication of contents with ease, even by just clicking on "share", are massive factors that increase the fecundity of the memes. A person who sees one of the contents about Tombili The Cat and who wants to share it with others can do so in a matter of seconds, without even having to leave his chair. Moreover, when sharing these contents on social media or transmitting them via emails, the person can add his own comments as well, and we may assume that this can trigger the desire to replicate the meme, thus increasing its fecundity.

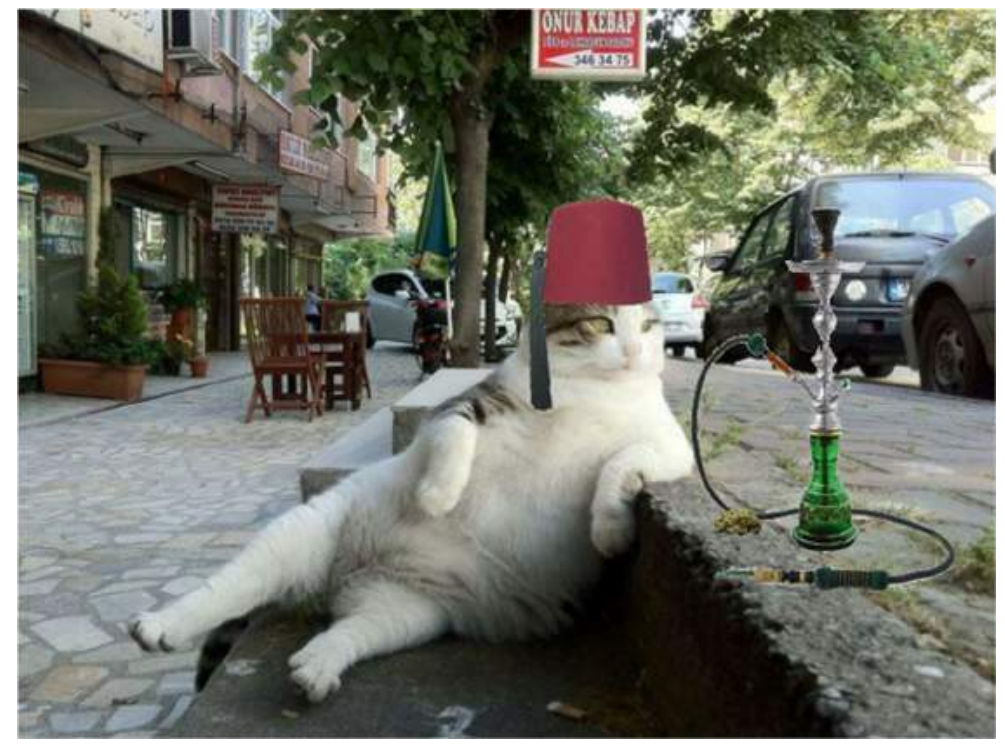

Image 4 Tombili the Cat

\section{Copying Fidelity}

Dawkins stated that longevity, fecundity and copying-fidelity are the qualities which provide a meme high survival value. Dawkins mentioned in his early writings on memes, that copying fidelity could alter in the case of memes since these memes go through a mutation and change when being transmitted. As mentioned above, in the case of the Internet memes, mutation is considered as a prerequisite for a content to be defined as an Internet meme. The photograph that first introduced Tombili The Cat to us have spread on the Internet in the form of various different image macros that had been edited and re-edited numerous times. The dissemination of the meme occurred through these image macros.

\section{Tombili The Cat and The Four Stages of Meme Replication}

For Tombili The Cat and all the other Internet memes, to be replicated means to be shared. All potential replication methods of memes are conducted by the developed opportunities digital communication offers. And without a doubt this situation causes alterations and transformations regarding Heylighen's meme replication processes. 


\section{Assimilation}

Many researches on the Internet culture agree that cat-related content has high viewing and high sharing ratios as well as receiving the most engagement (url6, 2019). This can be counted as a significant factor in the case of Tombili. In Tombili's case, the global love for cats is accompanied by a more local interest, the street culture. In the interviews done by the residents of the neighborhood, it is apparent that the street cat Tombili was regarded as an individual, an important character among the locals. (url7, 2019) It is possible to assume that the sympathy felt for Tombili had an influence on the replication process of the meme. Another point that should be considered while analyzing the reasons behind Tombili's success in assimilation i.e., being noticed, understood and accepted is Tombili's posing almost like a human being lounging on a chair. Various research has shown that anthropomorphism - attribution of human traits to non-human entities - have always been interesting for humans (Miltner, 2012). It is also important to remember that Internet memes related to cats, especially the LOLCat phenomenon, have become such a dominant mainstream pop element in 2000's that it even created its own language (Tekrin, 2014). To sum up, in the process of meme replication Tombili survives the assimilation test successfully with the help of a mysterious, yet strong bond between street culture, cats and the Internet.

\section{Retention}

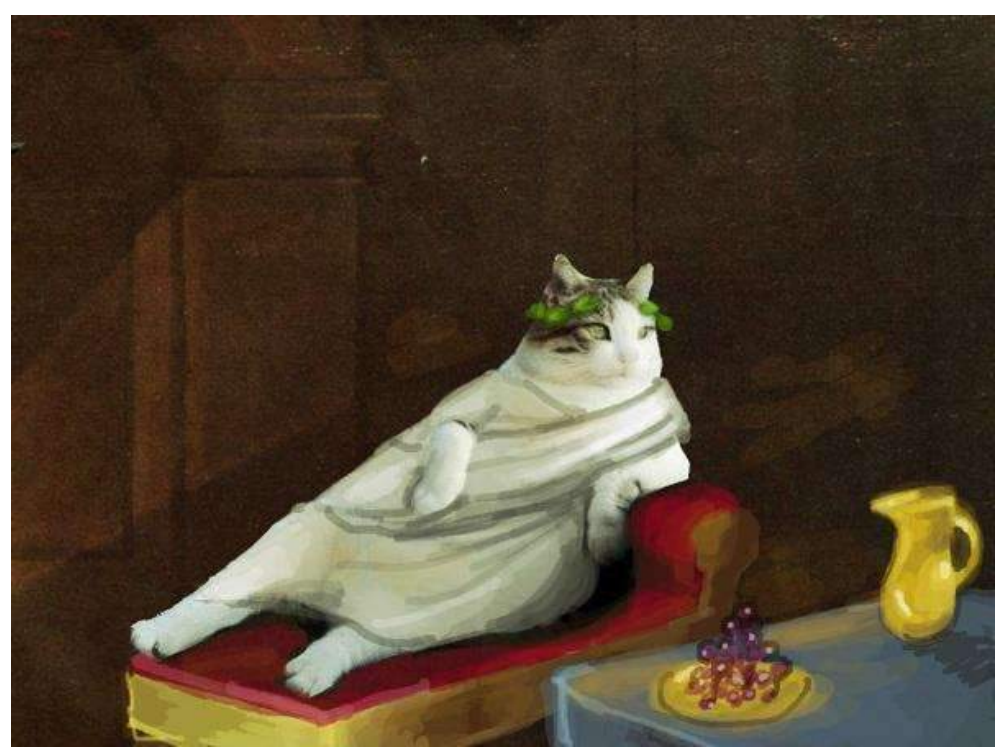

Image 5. Tombili the Cat

Tombili's fate has an undeniable influence on the second stage of Heylighen's model, that is retention. His sudden death, the petition to build a statue in his name, the ceremony of placing the statue, the stealing of the statue were all newsworthy events which took place in digital press and social media. This in turn, can be seen as a factor that made it possible for the Tombili meme to remain in people's minds for a considerable length of time. The digital environment, especially social media in which the meme was shared also has a great role in the process of retention. Just as it is the case in Dawkins' longevity criteria, the meme takes place in many people's feed every time the copies of it are shared and this has a positive influence on its retention. While many people may be seeing the meme for the first time, those who have seen it before come across the meme again and again, its potential of retention increases.

\section{Expression}

The third stage of replication, expression, requires the meme to be considered valuable enough to be transmitted to others. In this case, a cute and funny cat such as Tombili gains a major advantage. First of all, Tombili is a meme that can attract the attention of people from every segment of society; it will not be associated with any political view or sociocultural class, and the person will not receive any negative reaction upon sharing it. At the same time people who share an image macro of 
Tombili end up sharing a meme which belongs to the amusing content category (see research showing that content with positive emotions has the potential to be shared more, Berger,J. and Milkman, K. 2012). Funny content receives a lot of interest on social media, that has big potential of being shared again and receiving positive engagement. However, may be of utmost importance, Internet memes provide an opportunity of expression which have never seen before. Contents in the form of image macros, including the examples of Tombili The Cat, can be edited in just seconds in the web platforms dedicated to internet memes such as Knowyourmeme and Memegenerator. This provides the user a chance of remixing and reproducing the content. A new text can be added to the image macro, the image can be edited or the background of the image can be altered completely. These practical editing opportunities are available for most digital Internet meme formats just as they are for image macros. Thus, individuals can express the memes in their own words.

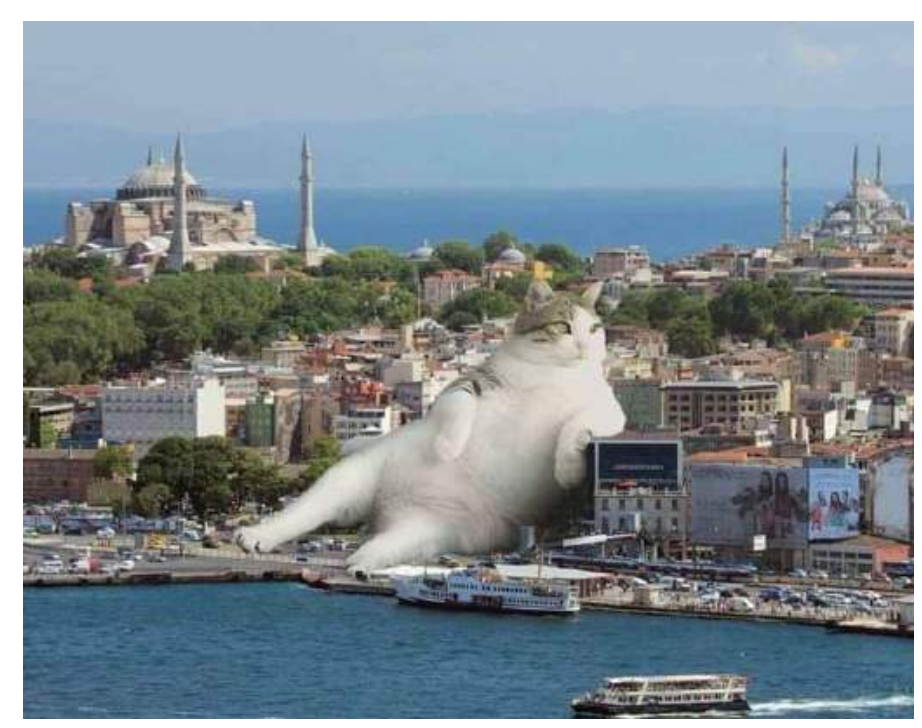

Image 6. Tombili the Cat

\section{Transmission}

The last stage of the replication process involves having the proper medium and using it sufficiently in order to transmit the expressed meme. It should be underlined that the transmission of the Tombili The Cat meme was conducted more practically via digital communication channels as opposed to analogue meme tools. Even thinking about how much faster it would be to transmit a photo or a cartoon of Tombili on a social media account than to have it in the newspaper gives an idea about this practice. In addition, it is necessary to consider that the copy is transmitted faster on the internet, hence are fewer possibilities for corruption and failure to reach the receiver.

\section{Conclusion}

In addition to emotional, social and cultural influences, we have seen that digital communication and the possibilities of the Internet are important factors in the spread of Tombili The Cat meme. However, these possibilities not only enabled the copying and dissemination of memes more effectively, but also enabled a transformation in memes.

Internet memes are copied and transferred very quickly thanks to the fastest data transmission means seen in the history of communication, moreover, it has been possible to edit them when they are replicated by means of digital technologies. As in the case of Tombili The Cat image macros, the internet meme can be easily edit by individuals and expressed again in the individual's own words and visual language. This has led to a major transformation in the copying fidelity of memes that have already been questioned since Dawkins, because Internet memes will only be able to remain as Internet memes for as long as this mutation continues. The biggest difference between the definition of a meme and an Internet meme lies there. 
Another major difference that has developed between memes and Internet memes is that social media platforms play an important role in the replication process of Internet memes. When Internet memes are shared- in other words replicated- on social media platforms, many potential hosts see the meme at the same time. Therefore, the first stage of the four stages of replication, the assimilation stage begins on numerous hosts simultaneously. Although a similar situation exists in mass media tools, there is no repetition factor in mass communication. On the other hand, as different people who are followed in a social media application share the same Internet meme, the potential host will encounter the same meme over and over again and this will increase the survival chance of the meme.

In light of all these findings, it is possible to argue that Internet memes are also memes, however they do not share the same properties with memes. Digital technologies have not only made memes spread more easily but also added new features to them. In this context it will be inadequate to claim that Internet memes are merely a digital version of memes. Internet memes are forms of memes that have gone under a digital transformation. Such as digital transformation means not only a digitalization of concepts and formats, but also a transformation, an alteration based on technology, the same can be argued for memes. Internet memes are a digital version of memes, however, digitalization has provided new tools and possibilities for memes and greatly accelerated the cultural evolution that was made by memes.

Every Internet meme can be considered as a meme but every meme cannot be considered as an Internet meme. Moreover, in some cases it may not be possible to say which memes are Internet memes and which are not. On one hand, the necessity and productivity of the Internet meme/meme distinction is debatable in current times where the world of the physical and online are becoming integrated every day. If the Internet didn't exist, Tombili The Cat could still be covered in foreign press, for example if he drew the attention of a foreign journalist. Maybe the residents of the neighborhood would again have a statue built for him, and if the statue were to be stolen it would still be newsworthy. However, none of these would have taken place with this speed, intensity and scale. Hundreds of people would not be able to edit the photo of Tombili and create their own versions to share with their friends. In short, Tombili could still be a meme but its influence and the scale of its dissemination would be greatly reduced. Memetic science states that memes are units that conduct cultural evolution. In this case, Internet memes have accelerated the cultural evolution and increased its influence.

\section{References}

[1] Berger, J. ve Milkman, K. (2012). What Makes Online Content Viral? Journal of Marketing Research.159 p. 192-205

[2] Börzsei, L. (2013). Makes a Meme Instead: A Concise History of Internet Memes. NewMedia Studies Magazine7. Erişim: 20.05.2019, http://works.bepress.com/linda_borzsei/2/

[3] Blackmore, S. (2011). Meme Machine. Oxford Press

[4] Brodie, R.(2014). Virus of the Mind. Hay House Press

[5] Dawkins, R. (1976) The Selfish Gene. [Elektronik Sürüm] Oxford. Erişim: 20.05.2019 https://archive.org/details/TheSelfishGene

[6] Goriunova, O., (2013). The force of digital aesthetics: on memes, hacking, and individuation. Zeitschrift fur Medienwissenschaft, 8, Editors: Erich Horl, Mark Hansen, "Medienasthetik".

[7] Heylighen, F. (1999). What Makes A Meme Successful. in: Proc. 15th Congress on Cybernet ics. p.418-423

[8] Miltner, K.M. (2014). "There's no place for lulz on LOLCats": The role of genre, gender, and group identity in the interpretation and enjoyment of an Internet meme. First Monday, 19(8).

[9] Moritz, E. (1990). "Memetic Science: I- General Introduction". Journal of Ideas. 1(1). Erişim: 20.05.2019

[10] Tekrin, D. (2014). Cyber-Carnival. Unpublished Master Thesis

[11] url1: https://bit.ly/2IR3aef (March, 2019)

[12] url2: https://bit.ly/2kuJ3IC (March, 2019) 
Communication and Technology Congress - CTC 2019 (April 2019 - Turkey, Istanbul)

[13] url3: https://bit.ly/2kDGtKi (March, 2019)

[14] url4: https://bit.ly/2kgeUpY (March, 2019)

[15] url5: https://bit.ly/2poYWMD (March, 2019)

[16] url6: https://www.wired.com/2015/08/how-cats-took-over-the-internet/ (March, 2019)

[17] url7: https://www.sabah.com.tr/yasam/2016/08/21/iste-fenomen-kedi-tombilinin-hikyesi (March, 2019) 